\title{
O Desafio da Oferta de Cursos de Especialização em Atenção Básica da Universidade Aberta do Sistema Único de Saúde aos Profissionais dos Programas de Provimento
}

The Challenge of Offering Specialization Courses
in Primary Health Care in the Open University of
the Unified Health System for Professionals of the
Provision Programs

\author{
Alysson Feliciano Lemos \\ Francisco Eduardo de Campos $^{I}$ \\ Pedro Quintana Brito \\ Edinalva Neves Nascimento ${ }^{I}$ \\ Vinícius de Araújo Oliveiral
}

\section{PALAVRAS-CHAVE}

- Educação.

- Educação em Saúde.

- Especialização.

- Atenção Primária à Saúde.

- Política de Saúde.
A implantação de novas políticas públicas de provimento de profissionais de saúde para a atenção básica demandou a oferta de oportunidades educacionais. O objetivo é apresentar a iniciativa do Sistema Universidade Aberta do SUS (UNA-SUS) na oferta de cursos de especialização em atenção básica aos profissionais dos programas de provimento. Participaram das ofertas 15 instituições públicas de ensino superior do Sistema UNA-SUS. Foram estudantes 41.100 profissionais de todo o Brasil, entre eles, médicos, dentistas e enfermeiros. Foram analisados os dados provenientes da Plataforma Arouca do Sistema UNA-SUS, sendo selecionados como indicadores quantitativos para análise o número de profissionais que concluíram o curso, de profissionais aguardando ingresso no curso, de estudantes cursando, de estudantes falecidos, de estudantes inativados sem ingresso e de estudantes reprovados ou que abandonaram o curso. Foi realizada ainda a análise de 2163 Trabalhos de Conclusão de Curso (TCC) que estavam disponíveis no Acervo de Recursos Educacionais em Saúde do Sistema UNA-SUS entre os meses de maio e outubro de 2017. Foi possivel realizar uma análise quantitativa e qualitativa seguindo as orientações do pensamento hermenêutico-dialético. Os resultados mostraram que até o momento 20.437 profissionais dos programas de provimento concluíram os cursos de especialização em atenção básica e ainda há 8.201 cursando. Em relação ao TCC, observou-se que as doenças crônicas não transmissíveis, os tumores e os problemas relacionados à gravidez, parto e puerpério, assim como aspectos relacionados à saúde infantil e saúde do idoso foram os mais evidentes nos projetos dos profissionais. Concluiu-se que a utilização de estratégias pedagógicas inovadoras pelas instituições de ensino provocou a reflexão sobre a prática, a mudança no processo de trabalho e a troca de saberes e experiências. Os cursos ofertados prepararam os participantes dos programas de provimento para a atuação na atenção básica e demonstraram melhor conhecimento sobre o Sistema Único de Saúde e letramento digital, especialmente, os estrangeiros e os intercambistas. 


\section{KEY-WORDS}

- Education.

- Health Education.

- Specialization.

- Primary Health Care.

- Health Policy.

Recebido em: 21/8/2018

Aceito em: 17/9/2018

\section{ABSTRACT}

The implementation of new public policies in Brazil to make up the shortfall of primary health care workers requires the provision of educational opportunities as part of the core strategy. This study presents the case of the provision of specialization courses for professionals in primary health care through the Open University of the Unified Health System (UNA-SUS) in the context of two federal programs: Valuing Primary Care (Provab) and More Doctors (PMMB). The vacancies were offered by fifteen public higher education institutions of the UNA-SUS System. Forty-one thousand professionals from all over Brazil, including doctors, dentists and nurses, where enrolled as students. Quantitative data about the courses and students was collected from the Arouca Platform - the information system of UNA-SUS. The qualitative indicators selected for analysis were: the numbers of professionals waiting to enroll on the course, of students who died, of professionals who failed to enroll, and of students who enrolled, graduated and dropped out of the course. The students were also required to write a course conclusion work, and these are published in the UNA-SUS open access repository (Ares). A sample of 2163 course conclusion works where reviewed using the hermeneuticdialectic approach. Results: It was found that to date, 20,437 graduated from basic health care specialization courses and a further 8,201 are currently taking courses. The man themes chosen for the course conclusion work were: chronic non-communicable diseases, tumors and pregnancy, childbirth and the puerperium, children's health and health of the elderly. It was concluded that the use of innovative pedagogical strategies by educational institutions led the professionals to reflect on their practice, review their work processes, and sometimes change those processes, and a broader sharing of knowledge and experiences. It seems that the courses prepared the participants for their professional activities in primary health care, improved their specific knowledge about Brazil's Unified Health System, and provided digital literacy, especially for foreign students.

\section{INTRODUÇÃO}

A Estratégia Saúde da Família (ESF) cresceu em cobertura e em número de profissionais, chamando a atenção para a necessidade de formação de recursos humanos que pudessem exercer uma prática em harmonia com as diretrizes da $\mathrm{ESF}^{1}$.

Essa necessidade de formação ficou ainda mais evidente com o Projeto de Expansão e Consolidação da Saúde da Família (Proesf), ocorrido entre os anos de 2002 e 2009, em que houve ampliação exponencial do número de equipes e de profissionais. Nessa época, o Ministério da Saúde (MS) investiu recursos financeiros para estruturação das unidades, territorialização, controle social e atuação em equipes multiprofissionais ${ }^{2}$. Porém, era um desafio consolidar uma proposta de capacitação e de educação permanente para os profissionais que já atuavam nas equipes das unidades de saúde.

Naquele momento, nenhuma universidade pública brasileira seria capaz de responder isoladamente à demanda de formação daqueles profissionais. Nenhuma tinha, ao mesmo tempo, suficiência de infraestrutura tecnológica, docentes, pessoal de apoio técnico-administrativo e mecanismos ge- renciais internos adequados para iniciativas desse vulto a tão curto prazo. Então, essa insuficiência gerava a necessidade de cooperação entre as instituições de ensino superior ${ }^{3}$.

Uma iniciativa do MS foi organizar uma rede colaborativa de instituições educacionais que pudessem ofertar cursos de especialização em atenção básica em larga escala. A primeira experiência foi a Rede Multicêntrica de Apoio à Especialização em Saúde da Família nas Grandes Cidades (Rede Maes), que permitiu aproximar as instituições para uma discussão sobre o assunto. Posteriormente, a Rede Maes foi transformada em uma ação do Programa Mais Saúde, definida como Universidade Aberta do SUS (UNA-SUS) ${ }^{1}$.

A UNA-SUS foi lançada em 2008 pelo MS com o objetivo de apoiar projetos de cursos de especialização na modalidade a distância. Esta iniciativa visava criar condições para o funcionamento de uma rede colaborativa de instituições acadêmicas destinada a atender às necessidades de formação e educação permanente do SUS. Essa rede de integração funcionaria por meio do intercâmbio de experiências, compartilhamento de material instrucional, cooperação para desenvolvimento e 
implementação de novas tecnologias educacionais em saúde, rede compartilhada de apoio presencial ao processo de aprendizagem em serviço e intercâmbio de informações acadêmicas dos estudantes para certificação educacional compartilhada. Dessa forma, seria possível ofertar a cada trabalhador de saúde oportunidades de aprendizado, como material para autoinstrução, cursos livres e de atualização, cursos de aperfeiçoamento, especialização e, até mesmo, mestrados profissionais ${ }^{4}$.

Institucionalmente, o Sistema UNA-SUS foi lançado como um arranjo institucional especializado em educação a distância na saúde e estabelecido pelo Decreto no 7.385/2010 (Brasil, 2010) $)^{5}$ e pela Portaria Interministerial no 10 , de 11 de julho de 2013 (Brasil, 2013) 6 . É coordenado por meio da atuação conjunta da Secretaria de Gestão do Trabalho e da Educação na Saúde do MS (SGTES/MS) e da Fundação Oswaldo Cruz (Fiocruz). O Sistema tem como pilares de gestão do conhecimento a Plataforma Arouca, base de dados dos profissionais de saúde, atividades educacionais e beneficiários e o Acervo de Recursos Educacionais em Saúde (Ares), repositório público dos produtos educacionais do Sistema. À Fiocruz compete também exercer a Secretaria Executiva do Sistema (SE/UNA-SUS/Fiocruz), com funções de monitoramento, avaliação, cooperação técnica e desenvolvimento de tecnologias educacionais ${ }^{7}$.

Naquele momento premente de formação dos profissionais da ESF, a primeira ação foi identificar instituições acadêmicas com o compromisso de ofertar minimamente mil vagas para Especialização em Saúde da Família. Em um primeiro momento, a adesão se deu pela negociação direta da equipe da Secretaria de Gestão do Trabalho e da Educação em Saúde (SGTES/ MS) com cada universidade interessada. Essa cooperação resultou no estabelecimento de convênios com cinco instituições, com previsão de oferta de 5.200 vagas em cursos de especialização, distribuídas em até três entradas. Assim, iniciou-se efetivamente a primeira fase do Projeto Piloto de Cesf da UNA-SUS ${ }^{3-8}$.

Outras instituições mostraram interesse em produzir recursos educacionais e ofertar Cesf em larga escala e na modalidade educação a distância pela UNA-SUS. Assim, em 2009, 13 instituições apresentaram projetos de parceria à SGTES/ MS, dos quais nove foram considerados aptos do ponto de vista técnico e administrativo, propondo a formação de outros 17.200 trabalhadores (médicos, dentistas e enfermeiros) do SUS. As ações desencadeadas pelas universidades permitiram aos profissionais o acesso a todas as oportunidades de aprendizado produzidas com recursos públicos quantas vezes fossem necessárias, além do registro do seu percurso educacional na Plataforma Arouca ${ }^{4}$.

Os ministérios da Saúde e da Educação formularam políticas públicas de valorização dos profissionais da atenção bá- sica de saúde. Em 2011, instituíram o Programa de Valorização dos Profissionais da Atenção Básica (Provab), que permitiu a atuação de profissionais de saúde em diferentes locais do País 9 . Os profissionais do Provab deveriam, necessariamente, realizar um curso de especialização pela Rede UNA-SUS.

Em 2013, foi implementado o Projeto Mais Médicos para o Brasil (PMMB) para provisão de atenção básica em saúde em regiões do País com escassez e ausência de profissionais. As ações formativas dos médicos também seriam realizadas pela Rede UNA-SUS ${ }^{10}$

O PMMB é uma intervenção inovadora e corajosa que estremeceu, simultaneamente, o exercício profissional e a formação médica em graduação e residência. Entretanto, há dependências e reverberações que devem ser analisadas. Se houvesse uma graduação de boa qualidade e generalista, como preconizam as Diretrizes Curriculares Nacionais (DCN) para Medicina ${ }^{11}$, talvez não fosse preciso oferecer um curso de especialização.

Se o mercado distribuísse equitativamente os recursos em saúde e os médicos fossem formados segundo as intenções dos pensadores da educação médica, talvez este artigo não precisasse ser escrito. Ele é uma reflexão sobre um processo de especialização centrado na profissão médica, facilitando o arranjo para que estes estejam disponíveis nos recantos mais vulneráveis do País. Os cursos se encontram longe de preparar o requerido médico generalista preconizado pelas $\mathrm{DCN}^{11}$, e, assim, o arranjo seria a proposição de especializações como "solução emergencial" para induzir o que as graduações não conseguiram alcançar.

Com a implementação de novas políticas públicas de saúde e a emergente necessidade de formação dos profissionais, a SGTES e a Secretaria Executiva da UNA-SUS/Fiocruz selecionaram e habilitaram novas instituições para apoiar a oferta de cursos. Assim, em 2013, foram selecionadas outras 22 instituições de educação superior credenciadas pelo Ministério da Educação. Atualmente, a Rede UNA-SUS é constituída por 35 instituições habilitadas para ofertar cursos de educação permanente em saúde, na modalidade a distância, aos profissionais de saúde de todo o País ${ }^{4}$ (Figura 1).

Para estes cursos de especialização, é obrigatória a apresentação de um Trabalho de Conclusão de Curso (TCC). Mediante autorização, os TCC são publicados no Ares, acervo público de materiais, tecnologias e experiências educacionais, construído de forma colaborativa, de acesso livre pela rede mundial de computadores ${ }^{5}$

O monitoramento e a avaliação dos estudantes em cada curso são realizados por meio da Plataforma Arouca, sistema de informação onde são registrados os cursos de especializa- 


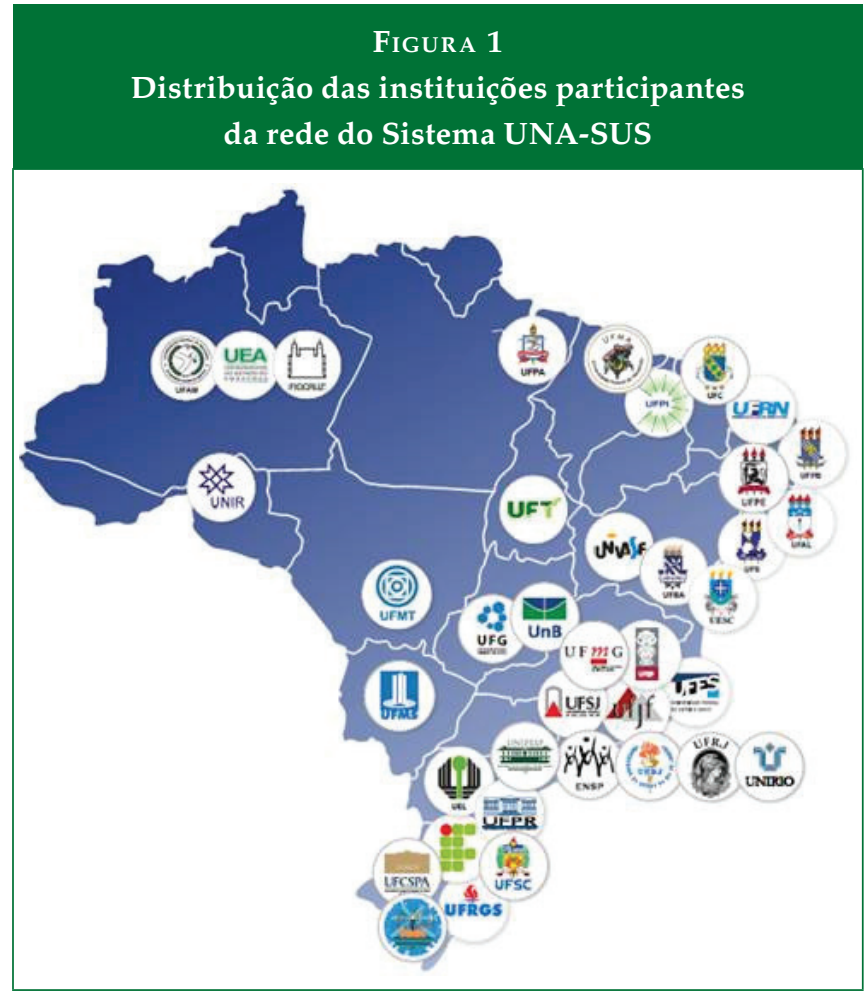

ção em atenção básica, suas ofertas educacionais, bem como as informações de situação dos estudantes ${ }^{5}$.

Portanto, pretende-se, neste estudo, apresentar a experiência do Sistema Universidade Aberta do SUS/UNA-SUS na oferta de cursos de especialização em atenção básica, modalidade a distância, aos profissionais participantes dos programas de provimento.

\section{O PERCURSO METODOLÓGICO}

$\mathrm{O}$ presente artigo respalda-se na perspectiva quali-quantitativa, de forma sistêmica e complementar, propiciando uma interpretação mais ampla do tema estudado ${ }^{12}$. Baseou-se no acesso e análise dos dados provenientes da Plataforma Arouca do Sistema UNA-SUS e revisão bibliográfica, pesquisa em artigos, legislação e demais fontes de informação necessárias ${ }^{13}$.

Foram levantados todos os artigos e demais publicações das instituições que compõem o Sistema UNA-SUS no que se refere aos cursos de especialização, assim como levantada e analisada toda a legislação relacionada à regulação das atividades educacionais dos programas de provimento do Ministério da Saúde.

Foram analisadas as informações de 41.100 profissionais integrantes dos programas de provimento Provab e PMMB, disponíveis na Plataforma Arouca, sendo selecionados como indicadores quantitativos para análise: número de profissio- nais que concluíram o curso; número de profissionais aguardando ingresso no curso; número de estudantes cursando; número de estudantes falecidos; número de estudantes inativados sem ingresso; número de estudantes reprovados ou que abandonaram o curso. Foi verificada a categoria profissional e a localização geográfica de cada estudante, bem como as instituições de ensino superior que ofertaram curso de especialização aos profissionais dos programas de provimento.

Graças à colaboração entre a UNA-SUS e a Organização Pan-Americana da Saúde (Opas/OMS) do Brasil ${ }^{14}$, foi possível realizar uma análise quantitativa e qualitativa de uma amostra de 2.163 TCC disponíveis no Ares, sendo 556 de participantes do Provab e 1.607 do PMM. Esta amostra foi analisada entre os meses de maio e outubro de 2017.

A análise qualitativa dos TCC seguiu as orientações do pensamento hermenêutico-dialético ${ }^{15}$. Foi realizada uma leitura exploratória de todo o material e, em seguida, uma análise na perspectiva interpretativa, buscando-se núcleos de sentido nos materiais descritos. Assim, as informações foram divididas em dois blocos, sendo um sobre objetivos de intervenção e outro acerca das áreas temáticas.

Quantos aos objetivos, os TCC foram classificados em: 1) promoção da saúde/prevenção de doenças; 2) melhoria da qualidade do cuidado; 3) organização e gestão dos serviços de saúde. Quanto ao teor das informações, foram organizados em 14 áreas temáticas: 1) alimentação e nutrição; 2) doenças transmissíveis; 3) hipertensão e diabetes; 4) modelos inadequados de atenção; 5) outras doenças crônicas; 6) saúde da criança; 7) saúde da mulher; 8) saúde do adolescente; 9) saúde do idoso; 10) saúde mental; 11) saúde sexual e reprodutiva; 12) tabagismo e outras drogas; 13) saúde bucal; 14) outros.

Buscou-se atender a todos os cuidados éticos referentes à preservação do anonimato dos participantes e das instituições do estudo.

\section{RESULTADOS DOS PROGRAMAS DE PROVIMENTO}

Os 41.100 profissionais dos programas de provimento estão localizados em todos os estados brasileiros, conforme demonstra a Figura 2. Os estados de São Paulo, Bahia e Ceará são os que apresentam maior quantidade de profissionais, diferentemente do Amapá, Roraima e Distrito Federal.

Os profissionais, em todas as regiões do País, atenderam aos pressupostos do Provab ${ }^{9}$ e do $\mathrm{PMMB}^{10}$, ou seja, o de prover médicos, dentistas e enfermeiros para atuarem na atenção básica em todo o País, especialmente nas áreas com maior necessidade. Os municípios são priorizados segundo a localização em regiões pobres e periferias de grandes cidades e os grupos populacionais indígenas, assentados rurais e quilombolas ${ }^{16}$. 


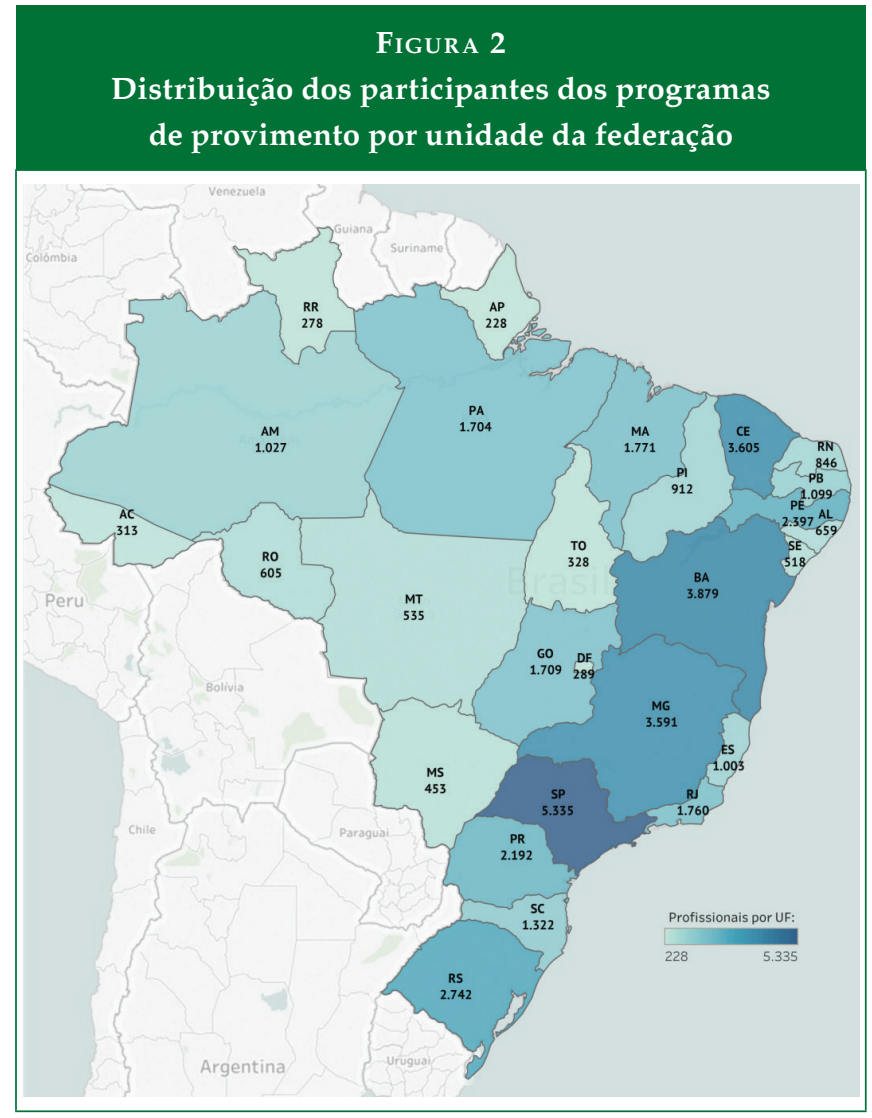

De 2013 a 2015, houve um incremento de 12.652 médicos no País, com ampliação significativa da cobertura de médicos em municípios localizados nas regiões Norte e Nordeste e de pequeno porte (especialmente entre 20 mil e 50 mil habitantes), bem como capitais e regiões metropolitanas ${ }^{17}$ (Tabela 1).

TABeLA 1

Distribuição dos profissionais dos programas de provimento por categoria profissional

\begin{tabular}{lcc} 
Categoria profissional & Total geral & $\%$ \\
\hline Dentista & 373 & 0,9 \\
\hline Enfermeiro & 941 & 2,3 \\
Médico & 39.786 & 96,8 \\
Total geral & 41.100 & 100
\end{tabular}

Os programas de provimento contemplaram dentistas, enfermeiros e médicos, sendo que estes últimos representaram $96,8 \%$ dos participantes. Isto diverge dos dados encontrados por Mattos et al. ${ }^{18}$, em que predominaram enfermeiros $(64,3 \%)$ e dentistas $(26,2 \%)$ em relação aos médicos $(9,5 \%)$, e dos dados encontrados por Oliveira et al. ${ }^{19}$, em que predominaram os enfermeiros $(26,4 \%)$.
Quando os enfermeiros e dentistas participaram dos programas de provimento por meio do Provab, tais profissionais eram os que mais realizaram os cursos de especialização em atenção básica. Posteriormente, com o PMMB, a especialização tornou-se específica para médicos, o que explica o quantitativo em destaque para esta categoria profissional.

É consensual a má distribuição de profissionais de saúde no mundo. Todos são possuidores do direito de ir e vir. Não é razoável, portanto, imaginar acorrentar os profissionais aos locais em que nasceram ou se graduaram. Assim, um verdadeiro carrossel forma-se entre os profissionais de saúde. Cubanos migram para a África do Sul, de onde profissionais se mudam para a Oceania, de onde outros vão para o Canadá rural, cujos trabalhadores se deslocam para áreas urbanas, e, destas, há pessoas que migram para o maior dos mercados em saúde, os Estados Unidos.

A expressão pejorativa white follows green dizia que aqueles que vestem aventais brancos andam atrás de cédulas verdes. Expressão similar foi usada no Brasil por Carlos Gentile de Mello, ainda antes do estabelecimento do SUS, a qual dizia haver uma não coincidente associação entre cidades que possuíam agências bancárias - leia-se dinheiro circulante - e médicos ${ }^{20}$.

Incontáveis políticas têm sido usadas no mundo para lidar com a má distribuição profissional, resumidas em documento publicado pela $\mathrm{OMS}^{21}$. Os incentivos financeiros não eram suficientes e, por mais que se incrementassem os salários, se limitava o recrutamento de profissionais, marginalizando ou anulando os ganhos em novos recrutamentos a partir de determinados níveis salariais.

Muitos brasileiros não tinham acesso à saúde, direito garantido constitucionalmente. Em 2013, o governo federal propôs o Provab voltado aos profissionais participantes das equipes de saúde da família, cujo principal atrativo era a bonificação aos médicos que servissem na atenção básica por um ou dois anos. A bonificação de $10 \%$ era um passaporte quase seguro para o ingresso aos programas de residência médica. Assim, muitos serviços de saúde contaram com médicos pela primeira vez.

O modus operandi do Provab foi matricular estes egressos (todos habilitados a exercer a profissão no País) em Cesf, o que possibilitava o pagamento de bolsa para trabalhador-estudante pelo Programa de Bolsas para a Educação pelo Trabalho ${ }^{22}$. No caso do PMMB, também foi realizado o pagamento de bolsa para o trabalhador ${ }^{23}$.

Os programas de provimento aumentaram a oferta profissional no mercado, permitindo que o Brasil chegasse próximo a parâmetros internacionais de mais de dois médicos por mil habitantes. 


\section{RESULTADOS DOS CURSOS DE ESPECIALIZAÇÃO}

Os programas de provimento preveem a formação dos profissionais por meio de diversas atividades de integração ensino-serviço. Cabe à rede de instituições da UNA-SUS ofertar a estes profissionais o curso de especialização e favorecer tal integração $0^{16}$. As atividades educacionais dos cursos devem desenvolver conhecimentos, habilidades e atitudes necessárias para o bom desempenho das funções, contribuindo, assim, para a garantia de qualidade da atenção à saúde prestada à população.

O Quadro 1 apresenta as 15 instituições de ensino superior do Sistema UNA-SUS envolvidas no processo educacional dos profissionais dos programas de provimento.

\begin{tabular}{lc}
\multicolumn{1}{c}{ QUADRo 1 } & Instituições de ensino superior do Sistema \\
& UNA-SUS que participaram das ações \\
& educacionais dos programas de provimento \\
\hline Instituição de ensino superior & Sigla \\
\hline Universidade do Estado do Rio de Janeiro & Uerj \\
\hline Universidade Federal de Alagoas & Ufal \\
\hline Universidade Federal da Bahia & UFBA \\
\hline Universidade Federal do Ceará & UFC \\
\hline Universidade Federal de Ciências da Saúde de Porto Alegre & UFCSPA \\
\hline Universidade Federal do Maranhão & UFMA \\
\hline Universidade Federal de Minas Gerais & UFMG \\
\hline Universidade Federal do Mato Grosso do Sul & UFMS \\
\hline Universidade Federal do Pará & UFPA \\
\hline Universidade Federal de Pernambuco & UFPE \\
\hline Universidade Federal de Pelotas & UFPel \\
\hline Universidade Federal do Piauí & UFPI \\
\hline Universidade Federal de Santa Catarina & UFSC \\
\hline Universidade de Brasília & UNB \\
\hline Universidade Federal de São Paulo & Unifesp \\
\hline
\end{tabular}

A Tabela 2 mostra a situação acadêmica dos profissionais. Observa-se até o momento a conclusão do curso de especialização por 20.437 profissionais dos programas de provimento, enquanto ainda há 8.201 cursando.

Além de solucionar a escassez de profissionais no País, é necessário garantir uma formação contínua e de qualidade, que promova reflexões e desenvolva a capacidade de resolução dos problemas reais.

A experiência acumulada do UNA-SUS demonstra que ofertas educacionais em grandes blocos podem se tornar monótonas e pouco atrativas. Os profissionais estão interessados em algo que lhes possa dar uma ajuda mais imediata, de forma a resolver os problemas com que se defrontam e que exi-

\begin{tabular}{|c|c|c|c|c|c|}
\hline & $\begin{array}{l}\text { Situação a } \\
\text { rogramas c } \\
\text { ensino st }\end{array}$ & $\begin{array}{r}\text { Tаве } \\
\text { cadêmica c } \\
\text { de provim } \\
\text { uperior do }\end{array}$ & $\begin{array}{l}\text { ELA } 2 \\
\text { dos profissio } \\
\text { ento por inst } \\
\text { Sistema UN }\end{array}$ & $\begin{array}{l}\text { onais dos } \\
\text { tituição de } \\
\text { JA-SUS }\end{array}$ & \\
\hline IES & Concluinte & Cursando & $\begin{array}{c}\text { Falecimento } \\
\text { do discente }\end{array}$ & $\begin{array}{c}\text { Reprovado } \\
\text { ou } \\
\text { abandono }\end{array}$ & $\begin{array}{l}\text { Total } \\
\text { geral }\end{array}$ \\
\hline Uerj & 1332 & 273 & & 385 & 1.990 \\
\hline Ufal & & 27 & & & 27 \\
\hline UFBA & 270 & & & 177 & 447 \\
\hline UFC & 2281 & 939 & 3 & 246 & 3.469 \\
\hline UFCSPA & 1463 & 718 & 1 & 974 & 3.156 \\
\hline UFMA & 2336 & 769 & & 11.805 & 4.290 \\
\hline UFMG & 2158 & 1060 & 1 & 839 & 4.058 \\
\hline UFMS & 1804 & 594 & 1 & 645 & 3.044 \\
\hline UFPA & & 350 & & & 350 \\
\hline UFPE & 1848 & 598 & & 568 & 3.014 \\
\hline UFPEL & 1716 & 2 & 1 & 1.180 & 2.899 \\
\hline UFPI & & 177 & & 1 & 178 \\
\hline UFSC & 1970 & 1383 & 2 & 163 & 3.518 \\
\hline UNB & 125 & & & 74 & 199 \\
\hline Unifesp & 3134 & 1311 & & 656 & 5.101 \\
\hline Profissior & hais aguardar & ndo matrícu & & & 4.573 \\
\hline $\begin{array}{l}\text { Profissior } \\
\text { matrícula }\end{array}$ & ais que forar & $m$ inativado & s antes da reali & ização de & 787 \\
\hline $\begin{array}{l}\text { Total } \\
\text { geral }\end{array}$ & 20.437 & 8.201 & 9 & 7.093 & 41.100 \\
\hline
\end{tabular}

gem soluções urgentes. A educação presencial não é suficiente para atender a uma realidade de mais de 5 mil municípios. $\mathrm{O}$ deslocamento de todos estes profissionais a centros mais tradicionais ou mesmo a cidades polo teria custado de centenas a milhares de vezes mais. Desta forma, a construção de uma infraestrutura que conhecesse as dificuldades e as tramas da educação a distância, compartilhando seus conhecimentos e recursos instrucionais produzidos em acervos abertos, representou um importante avanço.

Neste sentido, as instituições do Sistema UNA-SUS se organizaram internamente e superaram desafios institucionais e pedagógicos. Elas realizaram trabalhos multidisciplinares e promoveram integração entre os Departamentos de Medicina, Odontologia, Enfermagem, Farmácia, Educação, Educação Física, Design e Ciência da Informação, ao lado do envolvimento de profissionais com diferentes graduações e especialidades. Além do mais, elas estreitaram os laços com os serviços e superaram os modelos pedagógicos tradicionais ${ }^{24}$.

As instituições da Rede UNA-SUS buscaram implementar estratégias pedagógicas que favorecessem a aprendizagem significativa, por meio da utilização de metodologias ativas, 
reflexivas e problematizadoras, conforme sugerem a Política Nacional de Educação Permanente em Saúde ${ }^{25}$ e as diretrizes do Sistema UNA-SUS ${ }^{6}$.

Desde as primeiras ofertas, a UNA-SUS/UFMS/Fiocruz Pantanal utiliza metodologias ativas de aprendizagem com foco no planejamento dialogado com as políticas públicas de saúde no processo de trabalho e na troca de saberes ${ }^{26}$. A UNA-SUS/UFSC também utilizou propostas reflexivas para transformação da prática, centradas em um modelo de atenção integral ${ }^{27}$.

A UNA-SUS/UFPel elaborou mais de cem casos clínicos interativos com situações comuns em atenção primária à saúde, tendo como referência casos reais de diferentes grupos populacionais. Os depoimentos dos estudantes demonstraram que os casos clínicos interativos favoreceram o acesso às bibliografias de qualidade e a ampliação dos conhecimentos sobre os temas que são importantes para a prática profissional ${ }^{28}$.

As tecnologias mais recentes também foram utilizadas pela UNA-SUS/UFCSPA para motivar os profissionais-estudantes dos programas de provimento. Para promover um aprendizado mais interativo, a instituição trabalha com jogos educacionais e realidade virtual ${ }^{29}$. Além disso, simula o contexto de cidades fictícias de diferentes regiões do País. Os profissionais médicos, dentistas e enfermeiros vivenciaram esta estratégia pedagógica da UNA-SUS/UFCSPA durante a realização do curso de especialização e, como resultado, observou-se baixa taxa de evasão na oferta dos $\operatorname{cursos}^{30}$.

Percebeu-se por meio deste estudo que as metodologias ativas se mostraram fundamentais nos cursos de especialização, pois foram capazes de promover uma aprendizagem significativa aos profissionais dos programas de provimento. Mesmo sabendo que as instituições da Rede buscam incorporar estas estratégias em suas ações pedagógicas, a Secretaria Executiva da UNA-SUS inseriu esta temática entre as ações de cooperação técnica com a Rede UNA-SUS, para fortalecer esta ação. Assim, no dia 2 de junho de 2017, com a colaboração da UNA-SUS/Fiocruz/UFMS, promoveu o webinário "Metodologias Ativas de Aprendizagem" para representantes de todas as instituições da Rede UNA-SUS ${ }^{31}$.

Em razão do uso de tecnologias, é cada vez mais possível criar estratégias educacionais interativas e coerentes com as realidades dos profissionais de saúde. Ademais, é viável ainda monitorar e rastrear a participação e o desempenho dos estudantes e dos tutores nos cursos de especialização ofertados, de forma rápida e virtual ${ }^{32}$.

Uma pesquisa qualitativa realizada pela UFCSPA referente aos cursos ofertados para médicos, enfermeiros e dentistas do Rio Grande do Sul mostrou motivação para realização do curso, mesmo sendo uma exigência do programa. Os cursos permitiram compartilhar saberes, trocar experiências e aprofundar os conhecimentos em saúde da família. Além disso, houve ampliação da visão e conscientização do processo de trabalho na equipe, aquisição de novas ferramentas de trabalho, organização da agenda e discussão de saberes nos núcleos profissionais e entre os núcleos da atenção primária à saúde, com base no princípio da integralidade ${ }^{18}$.

Outro estudo qualitativo realizado com médicos, dentistas e enfermeiros egressos do curso de especialização da UNA-SUS/Unifesp demonstrou uma visão ampliada das necessidades de saúde da população e do estabelecimento de vínculo, o que é essencial ao processo de cuidado ampliado e inovador. Foi possível também reconhecer o papel e a importância dos diferentes profissionais da equipe, melhorar o processo de gestão por meio de decisões compartilhadas, organizar o processo de trabalho e considerar os dados epidemiológicos no planejamento das ações ${ }^{33}$.

\section{RESULTADOS DOS PROJETOS DE INTERVENÇÃO DOS TRABALHOS DE CONCLUSÃO DE CURSO}

A análise do relatório da Opas ${ }^{14}$ permitiu verificar que os TCC dos profissionais contemplam diferentes temáticas nos projetos de intervenção (Tabela 3).

\begin{tabular}{|c|c|c|}
\hline \multicolumn{3}{|c|}{$\begin{array}{c}\text { Áreas temáticas dos Projetos de Intervenção } \\
\text { dos Trabalhos de Conclusão de Curso dos } \\
\text { profissionais dos programas de provimento }\end{array}$} \\
\hline Áreas temáticas & f & $\%$ \\
\hline Alimentação e nutrição & 45 & 2,1 \\
\hline Doenças transmissíveis & 81 & 3,7 \\
\hline Hipertensão e diabetes & 961 & 44,4 \\
\hline Modelos inadequados de atenção & 96 & 4,4 \\
\hline Outras doenças crônicas & 35 & 1,6 \\
\hline Saúde da criança & 147 & 6,8 \\
\hline Saúde da mulher & 158 & 7,3 \\
\hline Saúde do adolescente & 89 & 4,1 \\
\hline Saúde do idoso & 142 & 6,6 \\
\hline Saúde mental & 47 & 2,2 \\
\hline Saúde sexual e reprodutiva & 210 & 9,7 \\
\hline Tabagismo e outras drogas & 93 & 4,3 \\
\hline Outros & 42 & 1,9 \\
\hline Saúde bucal & 17 & 0,8 \\
\hline Total & 2.163 & 100 \\
\hline
\end{tabular}

As doenças crônicas não transmissíveis, os tumores e os problemas relacionados a gravidez, parto e puerpério, assim como aspectos relacionados à saúde infantil e saúde do idoso foram os mais evidentes nos projetos dos profissionais. A aná- 
lise das áreas temáticas selecionadas para intervenção no TCC mostra que há coerência com as principais doenças e causas de morte no Brasil ${ }^{34}$.

Houve destaque para hipertensão e diabetes", corroborando os dados encontrados na intervenção realizada pelos médicos que cursaram a especialização da UNA-SUS/UFPel. Entre os 1.180 médicos que concluíram o curso, 433 (44,2\%) realizaram intervenções em pessoas com diagnóstico de hipertensão e/ou diabetes; 176 (18\%) em ações do pré-natal e puerpério; 149 (15,2\%) na área da prevenção do câncer de colo de útero e mama; 119 (12,2\%) em saúde do idoso; e 102 (10,4\%) em saúde da criança ${ }^{35}$.

A análise de 1.204 TCC realizada pela Unasus/UFSC mostrou que os estudantes-profissionais do PMMB dos estados do Paraná e de Santa Catarina propuseram projetos de intervenção especialmente com “doenças crônicas" (68,11\%), destacando-se hipertensão arterial sistêmica $(48,67 \%)$ e diabetes mellitus (8,06\%). Identificaram-se outras temáticas, como, por exemplo, saúde da mulher $(11,38 \%)$, saúde mental $(5,81 \%)$, doenças infecciosas e parasitárias (4,24\%), e tabaco, álcool e outras drogas $(3,65 \%)^{27}$.

As áreas temáticas demonstram a necessidade de fortalecer as competências clínicas e de saúde pública para enfrentar os problemas de saúde prevalentes e de alta complexidade, assim como a dificuldade em seu tratamento integral, tais como: doenças crônicas não transmissíveis e degenerativas, especialmente hipertensão e diabetes mellitus; tumores malignos, com especial atenção ao câncer do colo do útero e de mama nas mulheres; câncer de próstata e pulmão nos homens; alcoolismo, tabagismo e outros vícios, como o vício em benzodiazepínicos; problemas de saúde mental, como depressão e ansiedade; gravidez na adolescência; saúde dos idosos, não apenas os problemas de saúde, mas também as condições de vida desfavoráveis.

A análise dos objetivos dos TCC permitiu identificar que a promoção da saúde/prevenção de doenças, a melhoria da qualidade do cuidado e a organização e gestão dos serviços de saúde foram os enfoques de intervenção prevalentes. A reorganização dos processos de trabalho foi a menos frequente, sendo necessários novos estudos que verifiquem esta informação.

De forma geral, os projetos pedagógicos das universidades contribuíram com os cursos de especialização, atendendo aos princípios, diretrizes e metodologias da atual Educação Permanente em Saúde (EPS), conforme a Política Nacional de Educação Permanente ${ }^{25}$ e a Política Nacional de Atenção Básica $^{36}$. Tais cursos tiveram impacto positivo na reflexão dos participantes e no conhecimento de suas respectivas práticas profissionais na atenção básica.
A equipe da UNA-SUS/UFPel mostrou que a experiência educacional foi considerada benéfica, refletida em melhores práticas clínicas e maior conhecimento da organização do trabalho ao seu serviço. Os médicos referiram ter aplicado na prática diária os conhecimentos construídos no decorrer do curso, favorecendo a organização do processo de trabalho em relação ao cuidado e a decisões de condutas ${ }^{35}$.

Pesquisadores da UNA-SUS/UFCSPA verificaram que o curso também trouxe mudanças positivas na prática de profissionais do Provab, como a reorganização do processo de trabalho, implementação de atividades coletivas para atender à demanda espontânea e maior compreensão do processo de trabalho ${ }^{37}$.

Outro trabalho escrito por médicos cubanos que participaram do PMM aponta que as ofertas baseadas em tecnologias de informação e comunicação melhoraram a qualificação dos profissionais do SUS. O desenvolvimento de EPS expande o espaço de ações oferecidas pelas equipes de saúde e provoca mudança das práticas de cuidados de saúde e organização do processo de trabalho ${ }^{38}$.

\section{CONCLUSÃO}

A oferta de curso de especialização em atenção básica pelo Sistema UNA-SUS permitiu a qualificação de profissionais de nível superior que atuam na atenção básica. Os cursos ofertados tiveram duplo efeito: preparar os participantes dos programas de provimento (é clara a inadequação dos graduados à realidade assistencial do País, mas é inútil no curto prazo saber de quem é a culpa) e permitir que as escolas médicas acumulem a experiência de educar para a atenção primária, gerando a possibilidade de que a experiência da especialização repercuta na reforma da graduação. Podem estar aí embriões para futuros departamentos de atenção básica ou outros arranjos transversais mais avançados.

Quanto a seu resultado imediato, a existência da especialização fez parte da arquitetura institucional que, ao engajar milhares de médicos na atenção primária, possibilitou o acesso a esta de milhões de brasileiros, que estariam alternativamente descobertos em relação à atenção à saúde, sendo que muitos destes viram pela primeira vez na vida consultórios funcionando perto de suas casas. Para os médicos estrangeiros e, especialmente, os intercambistas, abre-se a oportunidade de melhor conhecimento do SUS e melhor letramento digital.

As ofertas espontâneas existentes antes do Sistema UNA-SUS, por melhores que fossem as intenções, não teriam passado de poucas centenas de vagas ofertadas e tampouco ofertariam massivamente e com qualidade cursos de especialização aos profissionais participantes dos programas de provimento do MS. 
Tamanho desafio contraposto à escassez da oferta em atenção básica por parte das escolas médicas pôde propiciar, adicionalmente e ainda que de forma incipiente, o habitus de colaboração entre as universidades, para a utilização de acervo comum e potencialização de ofertas. Reconhecer que os recursos educacionais produzidos por outrem são de qualidade e, em contrapartida, disponibilizar os seus para uso comum fortalece as relações de colaboração e reciprocidade, sem as quais seria mais estreito e afunilado o universo da educação.

A reflexão sobre a realidade sanitária brasileira e sua proposta inclusiva de atenção básica como porta de entrada do SUS, contextualizada na concretude do dia a dia dos serviços, é propiciada por um curso em que se utilizam tecnologias de informação e comunicação. Isto seria impossível usando-se a educação presencial sem descontinuar a prestação de serviços. Criou-se um processo menos verticalizado, propiciando reflexão e intercâmbio de experiências, abrindo a possibilidade de que a educação não apenas certificasse os estudantes, mas contribuísse efetivamente para a mudança do processo de trabalho. É esperado que esta intervenção resulte em melhoria na atenção e na própria saúde da população, que constitui o objetivo desta intervenção.

\section{REFERÊNCIAS}

1. Campos FE, Haddad AE, Oliveira VA, Aguiar RAT. O desafio dos processos e do mercado de trabalho na APS- o desafio da formação e da qualificação. In: Brasil. Ministério da Saúde. Secretaria de Atenção à Saúde. Departamento de Atenção Básica. Memórias da saúde da família no Brasil. Brasília: Ministério da Saúde, 2010. 144 p.

2. Brasil. Ministério da Saúde. Secretaria de Atenção à Saúde. Departamento de Atenção Básica. PROESF - Projeto de Expansão e Consolidação do Saúde da Família. Brasília: Ministério da Saúde, 2010. 18p.

3. Oliveira VA. O quebra cabeça da Universidade Aberta do SUS. In: Gusmão CMG, Borba SASVR, Menezes Júnior JV, Oliveira CAP, Nascimento EN, Oliveira VA. Relatos de uso de tecnologias educacionais na educação permanente de profissionais de saúde no sistema Universidade Aberta do SUS. Recife: Editora Universitária UFPE; 2014. p. 10-27

4. Oliveira VA, Nascimento EN, Franco SM, Brasil LSB, Lobo LCG. Constituição e expansão da rede UNA-SUS: trajetória 2008 a 2015. In: Gusmão CMG, Borba VR, Menezes Júnior JV, Oliveira CAP, Nascimento EN, Oliveira VA. II Relato de experiências em tecnologias educacionais do Sistema UNA-SUS 2015. Recife: Editora Universitária UFPE; 2015. p.14-34.
5. Brasil. Decreto $n^{\circ} 7.385$, de 8 de dezembro de 2010. Institui o Sistema Universidade Aberta do Sistema Único de Saúde - UNA-SUS e dá outras providências. Diário Oficial da União, Poder Executivo, Brasília, DF, 9 dez. 2010. p. 1.

6. Brasil. Portaria Interministerial $\mathrm{n}^{\mathrm{o}} 10$, de 11 de julho de 2013. Regulamenta o Decreto $\mathrm{n}^{\mathrm{o}} 7.385$, de 8 de dezembro de 2010, que instituiu o Sistema Universidade Aberta do Sistema Único de Saúde (UNA-SUS). Diário Oficial da União, Poder Executivo, Brasília, DF, 15 jul. 2013. Seção 1, p. 123.

7. Campos FE, Lemos AF, Oliveira VA. UNA-SUS: um ecossistema resiliente. In: Campos FE, Lemos AF, Oliveira VA, Franco SM, Nascimento EN, Oliveira AEF, Reis RS, Garcia PT. Experiências exitosas da Rede UNA-SUS: trajetórias de fortalecimento e consolidação da Educação Permanente em Saúde no Brasil. São Luís: EDUFMA; 2017. p. 15-30.

8. Oliveira VA, Brasil LSB. Repensando a educação permanente em saúde na sociedade da informação: a experiência da Universidade Aberta do Sistema Único de Saúde. In: TRINDADE, M. A. B. (Org.). As tecnologias da Informação e Comunicação (TIC) no Desenvolvimento de Profissionais do Sistema Único de Saúde SUS. São Paulo: Instituto de Saúde, 2011. p. 191-217. 14-34.

9. Brasil. Ministério da Saúde. Gabinete do Ministro. Portaria Interministerial $n^{\circ}$ 2.087, de $1^{\circ}$ de setembro de 2011. Institui o Programa de Valorização do Profissional da Atenção Básica. 2011.

10. Brasil. Portaria interministerial no 1.369 , de 8 de julho de 2013. Dispõe sobre a implementação do Projeto Mais Médicos para o Brasil. Diário Oficial da União, Poder Executivo, Brasília, DF, 9 jul. 2013.

11. Brasil. Ministério da Educação. RESOLUÇÃO № 3, DE 20 DE JUNHO DE 2014. Institui Diretrizes Curriculares Nacionais do Curso de Graduação em Medicina e dá outras providências. Brasília: Ministério da Educação; 2014.

12. Minayo MCS, Sanches O. Quantitativo-qualitativo: oposição ou complementaridade? Cad Saúde Pública. 1993; 9(3): 239-262.

13. Gil AC. Métodos e técnicas de pesquisa social. 5a ed. São Paulo: Atlas; 1999

14. OPAS-UNASUS. Caracterización de las ofertas formativas para profesionales médicos del Programa Mais Médicos. Estudio exploratorio de los TCCs de los Cursos de Especialización en Salud de la Familia (PMM y PROVAB). Brasilia, Noviembre 2017

15. Gomes R, Souza ER, Minayo MCS, Malaquias JV, Silva CFR. Organização, processamento, análise e interpretação de dados: o desafio da triangulação. In: Minayo MCS, As- 
sis SG, Souza ER. organizadores. Avaliação por triangulação de métodos: abordagem de programas sociais. Rio de Janeiro: Fiocruz; 2005. p. 185-221.

16. Pinto HA, Oliveira FP, Santana JSS, Santos FOS, Araujo SQ, Figueiredo AM, et al. Programa Mais Médicos: avaliando a implantação do Eixo Provimento de 2013 a 2015. Interface (Botucatu). 2017; 21(Supl.1):1087-101.

17. Girardi SN, Stralen ACSV, Cella JN, Maas LWD, Carvalho CL, Faria EO. Impacto do Programa Mais Médicos na redução da escassez de médicos em Atenção Primária à Saúde. Ciência \& Saúde Coletiva. 2016; 21(9): 2675-84.

18. Mattos LB, Dahmer A, Magalhães CR. Contribuição do curso de especialização em Atenção Primária à Saúde à prática de profissionais da saúde. Rev. ABCS Health Sci 2015; 40(3):184-189.

19. Oliveira VA, Savassi LCM, Lemos AF, Campos F. Elearning for health in Brazil: UNA-SUS in numbers. Journal of the Internacional Society for Telemedicine and Ehealth. 2016; 4(e9): 1-7.

20. Campos FE, Machado MH, Girardi SN. A fixação de profissionais de saúde em regiões de necessidades. Divulgação em Saúde para Debate. 2009; 44: 13-24.

21. World Health Organization. Increasing access to health workers in remote and rural areas through improved retention: global policy recommendations. 2010.

22. BRASIL. Presidência da República. Casa Civil. Subchefia para Assuntos Jurídicos. Lei $n^{\circ} 11.129$, de 30 de junho de 2005. Institui o Programa Nacional de Inclusão de Jovens ProJovem; cria o Conselho nacional da Juventude- CNJ e a Secretaria Nacional de Juventude; altera as Leis $n^{\circ} \mathrm{s} 10.683$, de 28 de maio de 2003, e 10.429, de 24 de abril de 2002; e dá outras providências. Portal da Legislação, Brasília, dez. 2017. Disponível em: <http://www.planalto.gov.br/ccivil_03/_ato2004-2006/2005/lei/111129.htm >. Acesso em: 26 dez. 2017.

23. BRASIL. Presidência da República. Casa Civil. Subchefia para Assuntos Jurídicos. Lei ${ }^{\circ} 12.871$, de 22 de outubro de 2013. Institui o Programa Mais Médicos, altera as Leis $\mathrm{n}^{\circ}$ 8.745, de 9 de dezembro de 1993, e nº 6.932, de 7 de julho de 1981, e dá outras providências. Portal da Legislação, Brasília, dez. 2017. Disponível em: < http:/ / www.planalto.gov. br/ccivil_03/_ato2011-2014/2013/lei/112871.htm >. Acesso em: 26 dez. 2017.

24. Carvalho RA, Struchiner M. Knowledge and expertise from traditional universities for the development of Open University of Brazilian National Health System (UNA-SUS) distance courses. Interface (Botucatu). 2017; 21(63):991-1003
25. Brasil. Ministério da Saúde. Gabinete do Ministro. Portaria n. ${ }^{\mathrm{o}}$ 1.996, de 20 de agosto de 2007. Dispõe sobre as diretrizes para a implementação da Política Nacional de Educação Permanente em Saúde e dá outras providências. 2007.

26. Machado JFFP, Carli AD, Kodjaoglanian VL, Santos MLM. Educação Permanente no cotidiano da Atenção Básica no Mato Grosso do Sul. Saúde e Debate. 2015; 39 (104): 102113.

27. Coelho EBSC, Bolsoni CC, Warmling D, Lindner SR, Boing AF, Assis FB, Verdi MIM, Faust SB. Análise dos projetos de intervenção do programa Mais Médicos nos estados do Paraná e Santa Catarina.. In: Campos FE, Lemos AF, Oliveira VA, Franco SM, Nascimento EN, Oliveira AEF, Reis RS, Garcia PT. Experiências exitosas da Rede UNA-SUS: trajetórias de fortalecimento e consolidação da Educação Permanente em Saúde no Brasil. São Luís: EDUFMA; 2017. p. 251- 264.

28. Fassa AG, Fantinel EJ, Soares DC, Carret MLV, Linhares RS, Maagh SB, Pinto LR, Fassa MEG, Fachini LA. Qualificação da prática clínica através de casos clínicos interativos. In: Campos FE, Lemos AF, Oliveira VA, Franco SM, Nascimento EN, Oliveira AEF, Reis RS, Garcia PT. Experiências exitosas da Rede UNA-SUS: trajetórias de fortalecimento e consolidação da Educação Permanente em Saúde no Brasil. São Luís: EDUFMA; 2017. p. 175-188.

29. Portella FF, Tubelo RA, Zanatta EJ, Pinto MEB, Dahmer A. Experiência da UNA-SUS/UFCSPA no desenvolvimento de jogos educacionais. In: Campos FE, Lemos AF, Oliveira VA, Franco SM, Nascimento EN, Oliveira AEF, Reis RS, Garcia PT. Experiências exitosas da Rede UNA-SUS: trajetórias de fortalecimento e consolidação da Educação Permanente em Saúde no Brasil. São Luís: EDUFMA; 2017. p. 193-210.

30. Dahmer A, Tubelo RA, Pinheiro LB, Costa MR, Pinto MEB. Virtual cities as content environments in a family health postgraduate programme. Journal of the International Society for Telemedicine and Ehealth. 2016; 4(7). p. 1-6.

31. Nascimento ENN, Oliveira VA, Lemos AF. Webconferências com integrantes da Rede UNA-SUS: relato de experiência e avaliação dos participantes. In: Campos FE, Lemos AF, Oliveira VA, Franco SM, Nascimento EN, Oliveira AEF, Reis RS, Garcia PT. Experiências exitosas da Rede UNA-SUS: trajetórias de fortalecimento e consolidação da Educação Permanente em Saúde no Brasil. São Luís: EDUFMA; 2017. p. 31-44.

32. Rendeiro MMP, Dias PRV, Oliveira AEF, França RM. Lecture Notes on Information Theory Vol. 1, No. 1, March 2013: 44-47. 2013 
33. Marin MJS, Nascimento ENN, Tonhom SFR, Alves SBAD, Girotto MA, Otani MAP, Silva LCP. Formação na Modalidade a Distância pela Universidade Aberta do SUS: Estudo Qualitativo sobre o Impacto do Curso na Prática Profissional. Revista Brasileira de Educação Médica. 2017; 41(2): 201-9.

34. Brasil. Ministério da Saúde. Saúde Brasil 2014. Uma análise de situação de saúde e das causas externas. Brasília 2015.

35. Thumé E, Wachs LS, Soares MU, Cubas MR, Fassa MEG, Tomasi E, Fassa AG, Facchini LA. Reflexões dos médicos sobre o processo pessoal de aprendizagem e os significados da especialização à distância em saúde da família. Ciência \& Saúde Coletiva. 2016; 21(9):2807-2814.

36. Brasil. Ministério da Saúde. Secretaria de Atenção à Saúde. Departamento de Atenção Básica. Política Nacional de Atenção Básica. Brasília: Ministério da Saúde, 2012

37. Mattos LB, Dahmer A, Magalhães. Contribuição do curso de especialização em Atenção Primária à Saúde à prática de profissionais da saúde. ABCS Health Sci. 2015; 40 (3): 184-9.

38. González JEC, González OI. La educación a distancia como modalidad de estudio en el Proyecto Mais Médicos para Brasil. Rev. EDUMECENTRO. 2015; 7 (2):191-8.

\section{COLABORAÇÃO DOS AUTORES}

Todos os autores participaram das etapas de elaboração do trabalho.

\section{CONFLITO DE INTERESSES}

Não há

\section{ENDEREÇO PARA CORRESPONDÊNCIA}

Campus Universitário Darcy Ribeiro. Av. L3 Norte, s/n, Gleba A. Ed. Administrativo da Fiocruz Brasília, 2 andar, Asa Norte, Brasília/DF. CEP: 70.904-130. 